\title{
Mitocôndrias no cenário da resposta imune inata exercida por macrófagos
}

\author{
Mitochondria in the scenario of the innate immune response exerted by macrophages
}

Mitocondrias en el escenario de la respuesta inmune innata ejercida por macrófagos

Giovanna Rosa Degasperi, Maria Júlia Medrado Marques ${ }^{1}$, Aline Juncker ${ }^{1}$, Isabela Martin ${ }^{1}$.

\begin{abstract}
RESUMO
Objetivo: Revisar na literatura a participação das mitocôndrias na resposta imune inata exercida pelos macrófagos, a fim de auxiliar na melhor compreensão da atuação destas células em processos infecciosos e inflamatórios. Método: A revisão foi realizada a partir da análise de diversos artigos científicos, selecionando aqueles efetivamente ligados ao tema proposto. Resultado: O metabolismo é um componente essencial da função das células do sistema imunológico. A função dos macrófagos está diretamente correlacionada ao seu metabolismo. Macrófagos com fenótipo M1, apresentam um estado metabólico com predominância da glicólise exercendo atividade antitumoral e antimicrobicida, enquanto que a fosforilação oxidativa e a oxidação de ácidos graxos fazem parte do metabolismo dos macrófagos M2 que atuam controlando processos inflamatórios. Considerações Finais: O metabolismo mitocondrial pode direcionar o tipo de resposta imunológica exercida pelos macrófagos, desta forma, a modulação de rotas mitocondriais podem ser caracterizadas como alvo terapêuticos. A modulação das mitocôndrias de macrófagos pode contribuir para inibição de processos infecciosos ou inflamatórios e até mesmo para a supressão tumoral.
\end{abstract}

Palavras-chave: Mitocôndria, Macrófagos M1 e M2, Metabolismo, Sistema imune.

\begin{abstract}
Objective: To review in the literature the participation of mitochondria in the innate immune response exerted by macrophages, in order to help in better understanding the performance of these cells in infectious and inflammatory processes. Method: The review was carried out from the analysis of several scientific articles, selecting those effectively linked to the proposed theme. Results: Metabolism is an essential component of the function of the cells of the immune system. The function of macrophages is directly correlated to their metabolism. Macrophages with M1 phenotype, present a metabolic state with predominance of glycolysis exerting antitumor and antimicrobicidal activity, whereas oxidative phosphorylation and oxidation of fatty acids are part of the metabolism of M2 macrophages that act controlling inflammatory processes. Final considerations: Mitochondrial metabolism may direct the type of immune response exerted by macrophages, in this way, the modulation of mitochondrial routes can be characterized as therapeutic target. Modulation of macrophage mitochondria may contribute to inhibition of infectious or inflammatory processes and even to tumor suppression.
\end{abstract}

Keywords: Mitochondria, M1 and M2 Macrophages, Metabolism, Immune system.

1Pontifícia Universidade Católica de Campinas (PUC-Campinas), Campinas-São Paulo.
^E-mail: giovannadegasperi@puc-campinas.edu.br 


\section{RESUMEN}

Objetivo: Revisar en la literatura la participación de las mitocondrias en la respuesta inmune innata ejercida por los macrófagos, a fin de auxiliar en la mejor comprensión de la actuación de estas células en procesos infecciosos e inflamatorios. Método: La revisión fue realizada a partir del análisis de diversos artículos científicos, seleccionando aquellos efectivamente conectados al tema propuesto. Resultado: El metabolismo es un componente esencial de la función de las células del sistema inmunológico. La función de los macrófagos está directamente correlacionada con su metabolismo. Los macrófagos con fenotipo M1, presentan un estado metabólico con predominio de la glicólisis ejerciendo actividad antitumoral y antimicrobicida, mientras que la fosforilación oxidativa y la oxidación de ácidos grasos forman parte del metabolismo de los macrófagos M2 que actúan controlando procesos inflamatorios. Consideraciones finales: El metabolismo mitocondrial puede dirigir el tipo de respuesta inmunológica ejercida por los macrófagos, de esta forma, la modulación de rutas mitocondriales pueden ser caracterizadas como objetivo terapéutico. La modulación de las mitocondrias de macrófagos puede contribuir a la inhibición de procesos infecciosos o inflamatorios e incluso para la supresión tumoral.

Palavras-clave: Mitocondrias, Macrófagos M1 y M2, Metabolismo, Immune system.

\section{INTRODUÇÃO}

O sistema imunológico inato tem um papel fundamental na resposta imune. Pesquisas recentes demonstraram que as mitocôndrias participam de diversas rotas de sinalização em células da imunidade inata, a exemplo dos macrófagos contribuindo para respostas efetivas (LIU PS e HO PC, 2018). Evidências crescentes sugerem que as mitocôndrias participam não somente da resposta imune viral, mas também facilitam a imunidade antibacteriana mediada por fagócitos, ao gerar espécies reativas de oxigênio (EROs) (KIM SJ et al., 2018; PRÓCHNICKI T e LATZ E, 2017). Portanto, além de seu papel já bem caracterizado no metabolismo celular e morte celular programada, as mitocôndrias parecem regular o sitema imune inato.

As mitocôndrias são organelas conhecidas por comandar o metabolismo do organismo. Como fonte de combustível geram o ATP através da fosforilação oxidativa usando piruvato (derivado da glicose e glicólise) e ácidos graxos (AGs), ambos oxidados no ciclo de Krebs. Também constituem fonte importante de espécies reativas de oxigênio (EROs), induzindo desta forma o estresse oxidativo em diversos contextos, dentre eles, na resposta à infecção bacteriana (BANOTH B e CASSEL SL, 2018).

EROs mitocondrial podem atuar nos macrófagos e junto a Nicotinamida-Adenina-Dinucleótido-Fosfato (NADPH) oxidase contribuir para a ampliação da produção de citocinas inflamatórias que são importantes para a destruição de microorganismos. Foi demonstrado que compostos como a metformina, capazes de inibir o complexo I mitocondrial (uma fonte de EROs) prejudicam a produção destas citocinas (KELLY B et al., 2015). Além disso, estudos evidenciam que lipopolissacarídeos (LPS) presentes em bactérias gram negativas foram capazes de ampliar a produção de EROs em macrófagos, a partir do reconhecimento do LPS via receptor Toll Like (WEST AP et al., 2011).

Recentemente, alterações complexas no metabolismo mitocondrial foram caracterizadas em macrófagos de modelos animais. Nos macrófagos ativados por LPS e IFN-y (macrófagos M1), há predominância da glicólise. Fazem parte do metabolismo de macrófagos ativados por IL-4 (macrófagos M2), a fosforilação oxidativa e a oxidação de ácidos graxos (EL KASMI KC et al., 2015).

Essas alterações metabólicas em resposta à natureza do estímulo estão se mostrando determinantes das funções efetoras dos macrófagos M1 e M2. Além disso, a reprogramação de macrófagos de M1 para M2 pode ser alcançada através da indução de alterações de pontos específicos de rotas metabólicas. Desta forma, descrevemos o papel que o metabolismo desempenha na função de macrófagos, influenciando desta forma a resposta imunológica, e apresentamos resultados encontrados em alguns estudos recentes que mostram que inibidores metabólicos podem ser uma nova abordagem terapêutica para o tratamento de doenças

REAS/EJCH | Vol. 11 (10) | e569 | DOI: https://doi.org/10.25248/reas.e569.2019 Página 2 de 7 
inflamatórias, uma vez que alteram vias de sinalização de células de sistema imune e, portanto o perfil de resposta imunológica (GANESHAN K e CHAWLA A, 2014; FORMENTINI L et al., 2017).

Diante do exposto, o objetivo desse estudo foi revisar na literatura científica, o conhecimento emergente sobre a atuação das mitocôndrias na resposta imune exercida pelos macrófagos, a partir da análise de artigos científicos, diretamente ligados ao tema proposto.

\section{MÉTODOS}

Trata-se de uma revisão da literatura acerca das mitocôndrias no cenário da resposta imune inata exercida por macrófagos. Os artigos correlacionados ao tema foram pesquisados e selecionados nas bases de dados SCIELO e PUBMED. Após a definição do objetivo, dados específicos de cada um deles correlacionados ao tema proposto foram extraídos para análise e apresentação na sessão de Resultados e Discussão. Os critérios de inclusão/exclusão para a redação do artigo foram: inclusão somente de artigos de revisão e originais; como estratégia de busca, utilização dos descritores macrófagos M1 e M2, mitocôndria e resposta imune inata, metabolismo de macrófagos, mitocôndria e macrófago; somente artigos de língua inglesa foram consultados; a seleção resultou em um total de 30 artigos. Também, foram considerados artigos dos anos de 2010 a 2019, sendo excluídos da análise os anteriores ao referido período.

\section{RESULTTADOS E DISCUSSÃO}

\section{Produção de energia e de espécies reativas de oxigênio durante a resposta imune inata exercida pelos macrófagos}

A mitocôndria possui importantes funções no organismo como a regulação da homeostase de cálcio, controle da morte celular, produção de EROs, além de exercer um papel central na produção de energia (VAKIFAHMETOGLU-NORBERG H et al., 2017). A produção de energia pela mitocôndria a partir de glicose e ácidos graxos ocorre através da fosforilação oxidativa, cujo componente central é a cadeia de transporte de elétrons (CTE). A CTE também é uma fonte de EROs (Figura 1).

A CTE consiste de uma série de complexos proteicos localizados na membrana mitocondrial interna. Os elétrons provenientes do Ciclo de Krebs são transportados pela CTE. Alterações conformacionais nos complexos proteicos permitem bombear prótons da matriz para o espaço intermembranas, gerando um gradiente eletroquímico conhecido como gradiente eletroquímico de prótons. Este gradiente é utilizado para direcionar a síntese de ATP pela ATP-sintase, que possibilita aos prótons fluírem a favor do seu gradiente eletroquímico. Esse movimento iônico é utilizado para direcionar produção de ATP, a partir de ADP e Pi (VAKIFAHMETOGLU-NORBERG $\mathrm{H}$ et al., 2017).

Os complexos I e III da CTE são importantes fontes de EROs. O complexo I (NADH: ubiquinona oxidoredutase) é um importante ponto de entrada de elétrons para a CTE em mitocôndrias. Ele oxida o NADH em $\mathrm{NAD}^{+}$, reduz a ubiquinona em ubiquinol e usa a diferença de potencial redox para translocar prótons através da membrana interna mitocondrial. A produção de EROs no complexo I é, portanto, dependente da relação NADH/NAD ${ }^{+}$(SAZANOV LA, 2007). A produção de EROS pela mitocôndria contribui para o estresse oxidativo celular. Entretanto, esta organela possui um importante sistema de defesa antioxidante que a protege de danos induzidos por EROS. Este sistema inclui a superóxido dismutase e tiorredoxinas redutase e a peroxidase.

Evidências recentes têm destacado a participação da mitocôndria na atividade ou comportamento de células do sistema imune inato. Tal participação pode ocorrer através do próprio metabolismo da mitocôndria que direciona a atividade das células do sistema imune inato ou por meio do envolvimento da organela em etapas de vias de sinalização destas células (BANOTH B e CASSEL SL, 2018; SANDIR R et al.,2017; LIU PS e HO PC, 2018). 
Figura 1 - Mitocôndria: Produção de energia e ATP.

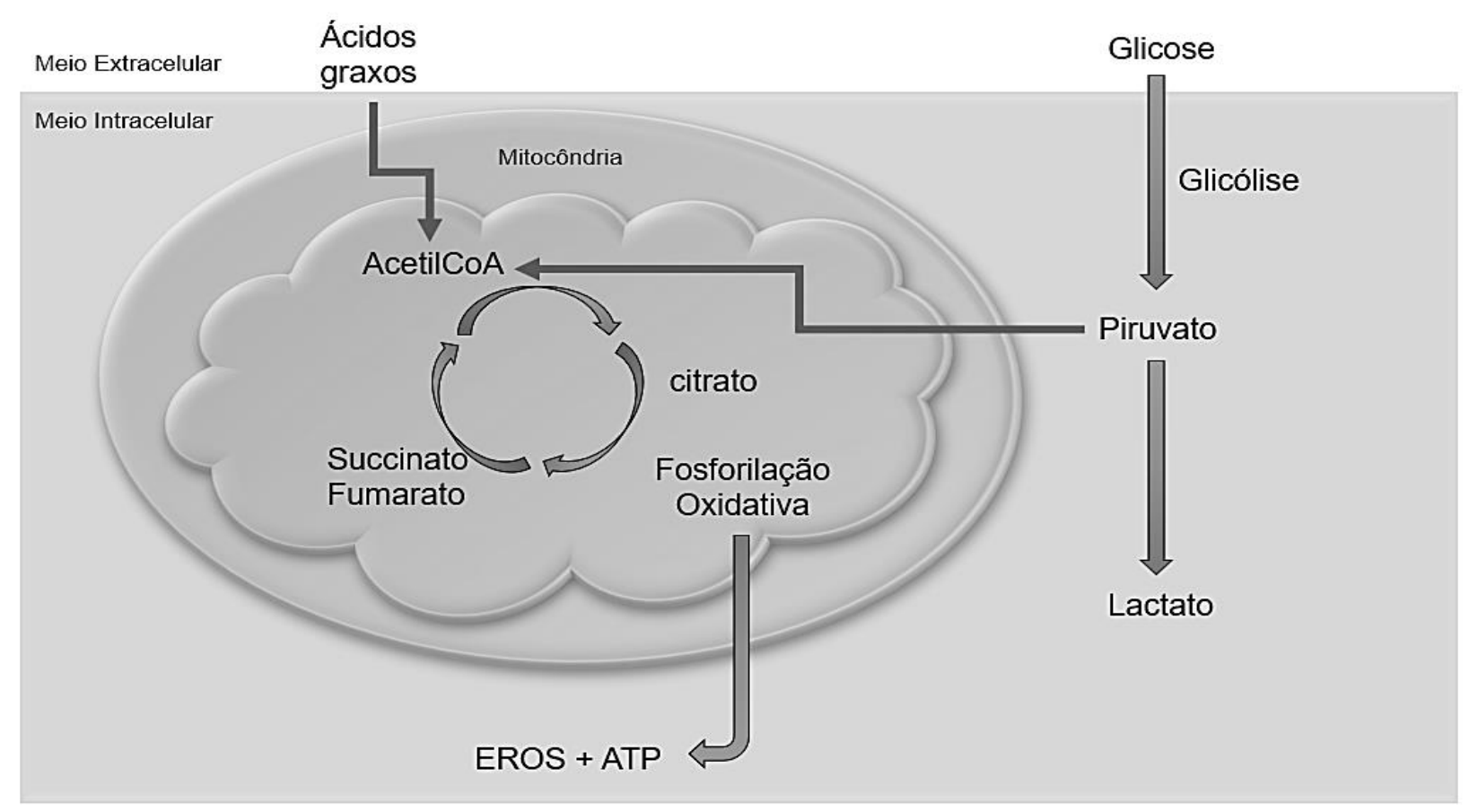

Fonte: Próprios autores, 2019.

Foi demonstrado que o LPS, um padrão molecular associado a patógeno (PAMP) presente em bactérias gram negativas, foi capaz de aumentar a produção de EROs mitocondrial. Neste caso, o reconhecimento do LPS pelo Toll Like (TLR), um receptor de reconhecimento padrão (RRP) presente também macrófagos, induziu recrutamento de um dos fatores associados ao receptor de TNF- $\alpha$, conhecido como TRAF6, o qual interage diretamente com complexo I da CTE, estimulando a produção de EROS (WEST AP et al., 2011).

EROs são componentes essenciais da resposta imune inata contra bactérias intracelulares e os fagócitos, a exemplo dos macrófagos, também são fonte destas espécies através da maquinaria da NADPH oxidase (nicotinamida adenina dinucleotídeo fosfato-oxidase do fagossoma). O fagossoma se forma quando ocorre a fagocitose, um remodelamento de membrana dos macrófagos para internalização e sequestro de microorganismo. O fagossomo torna-se um fagolisossomo, uma estrutura com a presença da NADPH oxidase e que oferece um meio ácido e hidrolítico especializado que tem a capacidade de degradar o microorganismo fagocitado (GRAY M e BOTELHO RJ, 2017; ZENG MY et al., 2019).

Estruturalmente, a NADPH oxidase é uma enzima composta de duas proteínas integrais de membrana, glicoproteína gp9 $1^{\text {phox }}$ e proteína adaptadora p22 ${ }^{\text {phox }}$, que juntas formam o flavocitocromo heterodimérico b558 considerado o núcleo da enzima, que transfere elétrons do NADPH para dentro da célula através da membrana e acoplando-os ao oxigênio molecular produz o ânion superóxido, um radical livre reativo (BELAMBRI SA et al., 2018; FILIP-CIUBOTARU F et al., 2016; THOMAS DC, 2017).

EROs mitocondriais podem também alcançar os fagossomos, desta forma, o burst oxidativo é ampliado. Nesta situação, há um estímulo da produção de citocinas inflamamatórias, destruição bacteriana e consequentemente controle da infecção (ABUIATA BH et al., 2018). Entretanto, a inibição da produção e EROs acarreta em prejuízo no controle de uma infecção. Estudos mostraram a produção de EROs induzida por LPS, bem como a produção de citocinas pró-inflamatórias foram prejudicados com a inibição do complexo I com a metformina, um composto antidiabético, ou com a rotenona, um inibidor do complexo I mitocondrial (KELLY B et al., 2015). Adicionalmente, a redução da produção destas citocinas também foi observada no tratamento com MitoQ, um sequestrador de EROs mitocondriais (DASHDORJ A et al., 2013). 


\section{As mitocôndrias direcionam o fenótipo e as funções dos macrófagos}

O metabolismo mitocondrial é determinante para os diferentes estados ou fenótipos adquiridos pelos macrófagos. Nas últimas décadas as pesquisas relacionadas ao metabolismo das mitocôndrias revelaram que estas organelas desempenham função imunorreguladora. Além de gerar espécies reativas de oxigênio, que possuem grande importância no metabolismo das células imunes, principalmente em funções antimicrobianas e pró-inflamatórias, a mitocôndria tem um papel fundamental na polarização e modulação da resposta de macrófagos (CHEN W et al., 2018; STOCKS CJ et al., 2018).

Os macrófagos apresentam um papel fundamental no sistema imune induzindo ou controlando resposta inflamatória, bem como na homeostase tecidual (DASHDORJ A et al., 2013). A inflamação começa quando estruturas encontradas na superfície de microrganismos, os chamados PAMPs ativam a resposta imune inata, por interação com os diferentes RRPs, a exemplo dos receptores TLRs nos macrófagos (VIJAY K, 2018). Após a interação de PAMPs com RRP, são desencadeadas múltiplas vias de sinalização, nas quais estão incluídos o fator nuclear kappa B (NF-kB) e as proteínas quinase ativadas por mitógenos (MAPK), os quais induzem a produção de citocinas pró-inflamatórias responsáveis por gerar uma resposta imune eficiente (TUR $J$ et al., 2017).

Em resposta a diversos sinais ambientais, os macrófagos caracterizam-se por dois estados de ativação denominados de macrófagos M1 ou classicamente ativados e macrófagos M2 ou alternativamente ativados (MILLS CD, 2015). O estado M1 surge quando os macrófagos são estimulados por LPS e TLR. Nesta condição, secretam citocinas pró inflamatórias, como TNF- $\alpha$, IL-6 e IL-1 $\beta$ e produzem EROs (CORRÊA DA SILVA F et al., 2018) (Figura 2). O fator de transcrição HIF-1 $\alpha$ é um dos principais fatores nucleares que contribui para o perfil metabólico de macrófagos M1. Em condições de hipóxia o HIF-1 $\alpha$ é expresso e reduz a atividade do Ciclo de Krebs e redução da respiração mitocondrial o que provoca um aumento na produção de EROs (SHI L et al., 2019). No estado M1, os macrófagos exercem atividades antimicrobicida e antitumoral (NA YR et al., 2018).

As citocinas da resposta imune do tipo Th2, IL-4 e IL-13, são determinantes para o estado M2, no qual os macrófagos ganham habilidade de expressão de receptores de manose e galactose, e ampliação da fagocitose. Macrófagos M2 atuam efetivamente atenuando a inflamação e promovendo reparo tecidual (Figura 2). A captação aumentada de glicose e a mudança para a glicólise são características-chave dos macrófagos $M 1$, enquanto a oxidação de ácidos graxos e a fosforilação oxidativa são as principais características metabólicas dos macrófagos M2. Ambas contribuem para as necessidades energéticas e para a regulação do rearranjo e fluidez de membrana que são essenciais para a fagocitose neste estado. Adicionalmente, estudos recentes sugerem que a glicólise também pode ser criticamente importante para a diferenciação de macrófagos M2 (WANG F et al., 2018).

Figura 2 - Polarização de macrófagos.

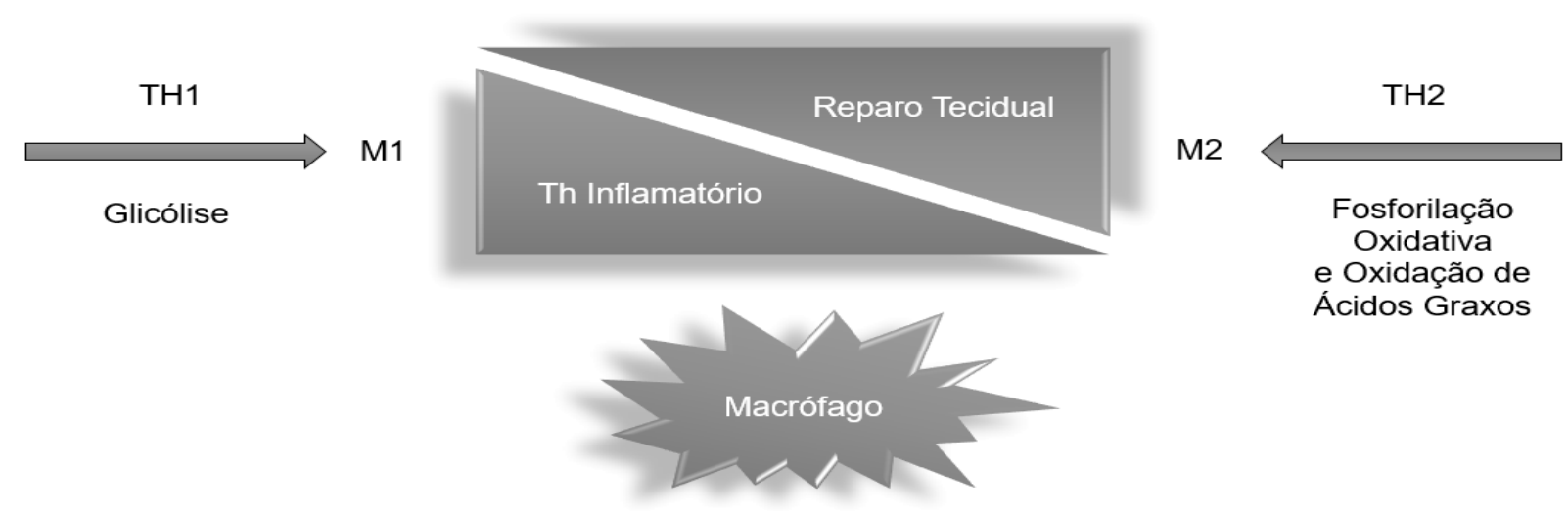

Fonte: Próprios autores, 2019. 
Também, foi demonstrado que a inibição da fosforilação oxidativa com oligomicina e o desacoplador FCCP, diminuíram a indução de arginase I e do receptor de manose e dectina 1 , ambos marcadores de macrófagos M2 (VATS D et al., 2006). Estudos evidenciaram ainda que a inibição da ATP sintase regula a polarização de macrófagos (FORMENTINI L et al., 2017; CHEN W et al, 2018). Foi observado um maior recrutamento e/ou polarização de macrófagos teciduais ao fenótipo anti-inflamatório M2 em resposta à colite induzida por dextran sulfato de sódio (DSS) em camundongos transgênicos expressando inibidor de ATP sintase IF1. Neste caso, a ATP sintase mitocondrial poderia representar um alvo relevante para promover a troca de classe (M1/M2) em macrófagos com fins terapêuticos em diversas doenças inflamatórias (FORMENTINI L et al., 2017).

\section{CONSIDERAÇÕES FINAIS}

Diversas foram as descobertas que evidenciam o envolvimento das mitocôndrias na determinação da resposta imune em células da imunidade inata como os macrófagos. Os estudos revelaram que o metabolismo mitocondrial pode direcionar o tipo de resposta imunológica exercida pelo macrófago durante uma infecção. Adicionalmente, foi demonstrado que a modulação da resposta imune de macrófagos pode ser induzida por inibidores de rotas mitocondriais. Desta forma, a organela pode representar um importante alvo terapêutico.

\section{REFERÊNCIAS}

1. LIU PS, HO PC. Mitochondria: A master regulator in macrophage and T cell immunity. Mitochondrion. 41:45-50, 2018. doi: 10.1016/j.mito.2017.11.002.

2. KIM SJ, AHN DG, SYED GH, et al. The essential role of mitochondrial dynamics in antiviral immunity. Mitochondrion. 41:21-27, 2018. doi: 10.1016/j.mito.2017.11.007.

3. PRÓCHNICKI T, LATZ E. Inflammasomes on the Crossroads of Innate Immune Recognition and Metabolic Control. Cell Metab. 26(1):71-93, 2017. doi: 10.1016/j.cmet.2017.06.018

4. BANOTH B, CASSEL SL. Mitochondria in innate immune signaling. Translational Research. 2018; 000:1-17. doi: 10.1016/j.trsl.2018.07.014.

5. KELLY B, TANNAHILL GM, MURPHY MP, et al. Metformin inhibits the production of reactive oxygen species from NADH: ubiquinone oxidoreductase to limit induction of IL-1beta, and boosts IL-10 in LPS-activated macrophages. J Biol Chem. 290: 20348-20359, 2015. doi: 10.1074/jbc.M115.662114.

6. WEST AP, BRODSKY IE, RAHNER C, et al. TLR signalling augments macrophage bactericidal activity through mitochondrial ROS. Nature. 472(7344):476-80, 2011. doi: 10.1038/nature09973.

7. EL KASMI KC, STENMARK KR. Contribution of metabolic reprogramming to macrophage plasticity and function. Semin Immunol. 27(4):267-75, 2015. doi: 10.1016/j.smim.2015.09.001.

8. GANESHAN K, CHAWLA A. Metabolic Regulation of Immune Responses. Annu Rev Immunol. 32:609-34, 2014. doi: 10.1146/annurev-immunol-032713-120236.

9. FORMENTINI L, SANTACATTERINA F, NÚÑEZ de ARENAS C, et al. Mitochondrial ROS Production Protects the Intestine from Inflammation through Functional M2 Macrophage Polarization Cell Rep. 19(6):1202-1213, 2017. doi: 10.1016/j.celrep.2017.04.036.

10. VAKIFAHMETOGLU-NORBERG H, OUCHIDA AT, NORBERG E. The role of mitochondria in metabolism and cell death. Biochemical and Biophysical Research Communications Biochem Biophys Res Commun. 482(3):426431, 2017. doi: 10.1016/j.bbrc.2016.11.088.

11. SAZANOV LA. Respiratory complex I: mechanistic and structural insights provided by the crystal structure of the hydrophilic domain. Biochemistry. 46(9):2275-88, 2007. doi: 10.1021/bi602508x. 
12. SANDHIR R, HALDER A, SUNKARIA A. Mitochondria as a centrally positioned hub in the innate immune response. Biochim Biophys Acta Mol Basis Dis.1863(5):1090-1097, 2017. doi: 10.1016/j.bbadis.2016.10.020.

13. LIU PS, HO PC. Mitochondria: A master regulator in macrophage and T cell immunity. Mitochondrion. 41:45-50, 2018. doi: 10.1016/j.mito.2017.11.002.

14. GRAY M, BOTELHO RJ. Phagocytosis: Hungry, Hungry Cells. Methods Mol Biol. 1519:1-16, 2017. Review.

15. ZENG MY, MIRALDA I, ARMSTRONG CL, et al. The roles of NADPH oxidase in modulating neutrophil effector responses. Mol Oral Microbiol. 2019 Jan doi: 10.1111/omi.12252.

16. BELAMBRI SA, ROLAS L, RAAD H, et al. NADPH oxidase activation in neutrophils: Role of the phosphorylation of its subunits. Eur J Clin Invest. 48 Suppl 2:e12951, 2018. doi: 10.1111/eci.12951.

17. FILIP-CIUBOTARU F, MANCIUC C, STOLERIU G, et al. NADPH OXIDASE: STRUCTURE AND ACTIVATION MECHANISMS (REVIEW). NOTE I. Rev Med Chir Soc Med Nat lasi. 120(1):29-33, 2016.

18. THOMAS DC. The phagocyte respiratory burst: Historical perspectives and recent advances. Immunol Lett. 192:88-96, 2017. doi: 10.1016/j.imlet.2017.08.016.

19. ABUAITA BH, SCHULTZ TL, O'RIORDAN MX. Mitochondria-Derived Vesicles Deliver Antimicrobial Reactive Oxygen Species to Control Phagosome-Localized Staphylococcus aureus. Cell Host Microbe. 24(5):625-636.e5, 2018. doi: 10.1016/j.chom.2018.10.005.

20. DASHDORJ A, JYOTHI, KR, LIM, et al. Mitochondria-targeted antioxidant MitoQ ameliorates experimental mouse colitis by suppressing NLRP3 inflammasome-mediated inflammatory cytokines. BMC Med. 11,178, 2013.

21. CHEN W, Sandoval H, Kubiak JZ, Li XC, Ghobrial RM, Kloc M. The phenotype of peritoneal mouse macrophages depends on the mitochondria and ATP/ADP homeostasis. Cell Immunol. 324:1-7, 2018. doi: 10.1016/j.cellimm.2017.11.003.

22. STOCKS CJ, SCHEMBRI MA, SWEET MJ, et al. For when bacterial infections persist: Toll-like receptor-inducible direct antimicrobial pathways in macrophages.J Leukoc Biol.103(1):35-51, 2018. doi: 10.1002/JLB.4RI0917358R.

23. VIJAY K. Toll-like receptors in immunity and inflammatory diseases: Past, present, and future. Int Immunopharmacol. 59:391-412, 2018. doi: 10.1016/j.intimp.2018.03.002. Erratum in: Int Immunopharmacol. Sep; 62:338, 2018.

24. TUR J, VICO T, LLOBERAS J, ZORZANO A, et al. Macrophages and Mitochondria: A Critical Interplay Between Metabolism, Signaling, and the Functional Activity. Adv Immunol. 133:1-36, 2017. doi: 10.1016/bs.ai.2016.12.001.

25. MILLS CD. Anatomy of a discovery: $\mathrm{m} 1$ and $\mathrm{m} 2$ macrophages. Front Immunol. 6:212, 2015. doi: 10.3389/fimmu.2015.00212. eCollection 2015.

26. CORREAA-DA-SILVA F, PEREIRA JAS, DE AGUIAR CF, et al. Mitoimmunity-when mitochondria dictates macrophage function. Cell Biol Int. 42(6):651-655, 2018. doi: 10.1002/cbin.10921.

27. SHI L, JIANG Q, BUSHKIN Y, et al. Biphasic Dynamics of Macrophage Immunometabolism during Mycobacterium tuberculosis Infection. MBio. 10(2), 2019. pii: e02550-18. doi: 10.1128/mBio.02550-18.

28. NA YR, JE S, SEOK SH. Metabolic features of macrophages in inflammatory diseases and cancer. Cancer Lett. 28; 413:46-58, 2018. doi: 10.1016/j.canlet.2017.10.044.

29. WANG F, ZHANG S, VUCKOVIC I, et al. Glycolytic Stimulation Is Not a Requirement for M2 Macrophage Differentiation. Cell Metab. 28(3):463-475.e4, 2018. doi: 10.1016/j.cmet.2018.08.012.

30. VATS D, MUKUNDAN L, ODEGAARD Jl, et al. Oxidative metabolism and PGC-1beta attenuate macrophagemediated inflammation. Cell Metab. 4(1):13-24, 2006. doi: 10.1016/j.cmet.2006.05.011. 